\title{
An investigation of using grey scale image analysis for predicting the amount of deposited electrospun nanofibres
}

\author{
A. H. Nurfaizey ${ }^{1,2 *}$, F. C. Long ${ }^{1}$, M. A. M. Daud ${ }^{1,2}$, N. Muhammad ${ }^{1,2}$, M. R. Mansor ${ }^{1,2}$ \\ and N. Tucker ${ }^{3}$ \\ ${ }^{1}$ Faculty of Mechanical Engineering, Universiti Teknikal Malaysia Melaka, \\ Hang Tuah Jaya, 76100 Durian Tunggal, Melaka, Malaysia \\ Phone: +6062346800 \\ *Email: nurfaizey@utem.edu.my \\ ${ }^{2}$ Centre for Advanced Research on Energy, Universiti Teknikal Malayia Melaka, \\ Hang Tuah Jaya, 76100 Durian Tunggal, Melaka, Malaysia \\ ${ }^{3}$ University of Lincoln, Brayford Pool, Lincoln, LND 7TS, United Kingdom
}

\begin{abstract}
When electrospinning, the amount of electrospun fibres deposited is difficult to determine due to the extremely small size and light weight of the fibres. Several methods have been used to predict the amount of deposited fibres including weighing, imaging and direct measurement. Yet, these methods have drawbacks that make them unsuitable for commercial scale process control. In this study, an image analysis method is used to predict the amount of deposited fibres over a significant area. When images of electrospun fibres are converted into grey scale images, it is suggested that the amount of fibres deposited can be predicted by measuring the grey scale intensity. Weighing method was used to validate the image analysis results. Weighing method was found wanting when the deposition time was short ( $p>0.05$ ) due to the insignificant fibre masses compared to the variation of substrates. The results suggest that image analysis method could be used to predict the amount of deposited electrospun nanofibres. Test on different polymers and substrates showed that the method was still capable to distinguish the samples. The developed method has the potential to be applied as an in-line non-destructive quality control method for electrospun fibre manufacture.
\end{abstract}

Keywords: Electrospinning; electrospun nanofibres; grey scale; image analysis.

\section{INTRODUCTION}

Electrospinning is a process where electrostatic forces are used to draw ultrafine polymeric fibres either from a polymer solution or melt [1]-[3]. The commercial process was first described in a patent filed by John Francis Cooley in 1900 [4], although it was previously described as a laboratory techniques by C.V. Boys in the 1860s. When a high voltage is applied to a pendant drop of a polymer solution, the polymer becomes highly electrified and charges accumulates on the surface of the droplet (Figure 1). As the electric forces overcomes the surface tension of the droplet, it is deformed into a conical shape known as Taylor cone 
[5], then the surface ruptures and a charged jet of polymer is ejected from the droplet. The jets initially move in a straight path before buckling (the so-called bending instability) and the motion towards the grounded collector continues in an expanding helical flight path. Along the flight path, solvent evaporation and fibre thinning occurs, continuing until the fibres land on the collector as a randomly oriented mat [6], [7].

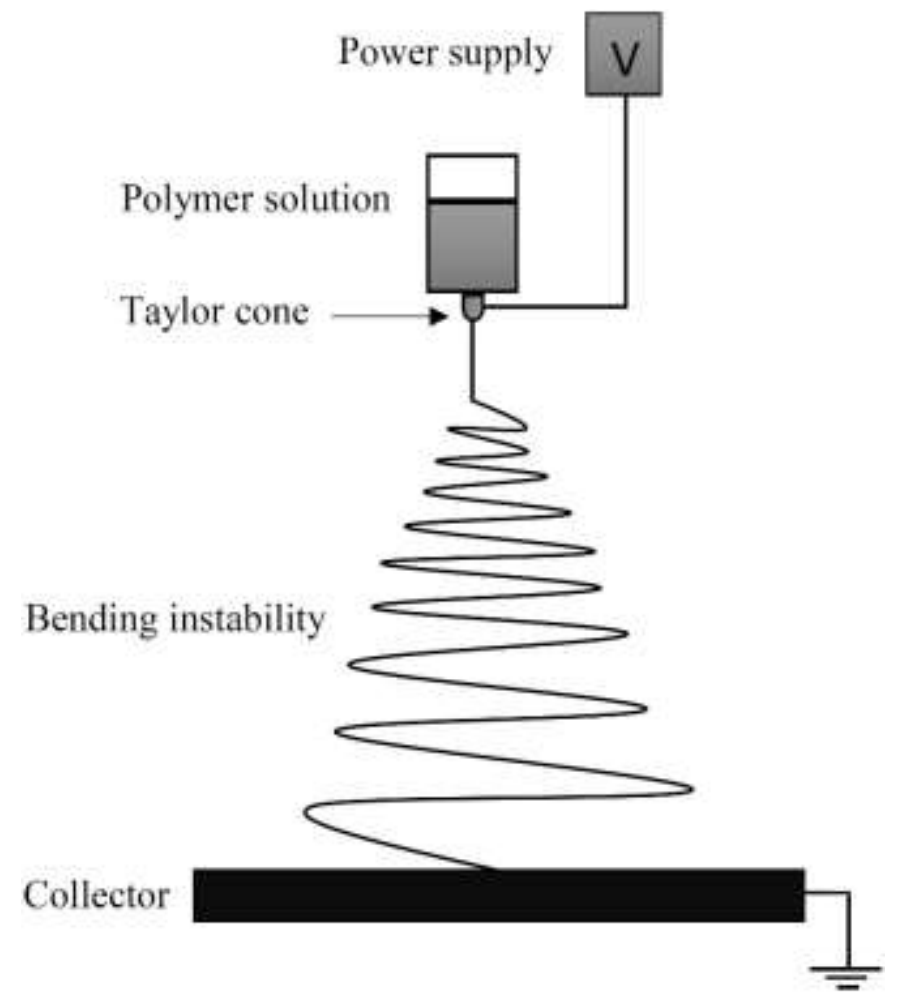

Figure 1. A schematic diagram of electrospinning process.

The characteristics of electrospun nanofibres structures such as high surface area to volume ration, light weight, small pore size and high permeability have attracted considerable amount of attention among researchers [3], [6]-[8]. The use of polymeric nanomaterials has been studied in various applications including filtrations, tissue engineering scaffolds, drug delivery systems, wound dressings, composite reinforcement, electronics and sensors [9][13].

In many applications, only a small amount of fibres are required to achieve significant improvement [14]-[16]. Gibson et al. [14] claimed that less than $1 \mathrm{~g} / \mathrm{m}^{2}$ of polyurethane electrospun nanofibres were required to capture most of aerosol particles that passing through a filtration media. S. Lee \& Obendorf [15] estimated that the penetration of pesticide mixtures through a filtration media can be reduced from $85 \%$ to $25 \%$ with the addition of $1 \mathrm{~g} / \mathrm{m}^{2}$ of polyurethane nanofibres. More recently, Li et al. [16] concluded that a small amount of nanofibres which equivalent to 10 seconds of deposition time was enough to increase the filtration efficiency of an aerosol filtration media from $17 \%$ to $92 \%$.

However, from production perspective, it is difficult to quantify the amount of deposited electrospun nanofibres due to their small sizes and light weight. The issue is further complicated when only a small amount of nanofibres are being investigated. For 
example, in a production that produces different grades of nanofibres products based on the amount of nanofibres, it is impossible to distinguish between the products using currently available techniques.

Several attempts to measure the thickness of deposited electrospun nanofibres have been reported. This includes direct measurement using a micrometer [17], analyses based on scanning electron microscopy images (SEM) [18] and light profilometry [17], [18]. However, there were certain drawbacks associated with all these methods. For example, SEM imaging requires a small cut samples to represent the whole product web, which as well as being destructive to the product and could also distort the structure of the fibres [19]. In addition, the SEM method is very time consuming and requires access to an SEM. Similarly, the light profilometry method requires handling of the small and possibly unrepresentative specimen which could damage the sample [20]. Direct measurement using a micrometer provides a quick and easy method of measuring thickness, however the applied forces as the results of direct contact with the sample which could crush or otherwise damage the sample. When the commercial process is considered, where a web of fibre typically $1.6 \mathrm{~m}$ wide is being continuously produced, any method that requires a sample to be cut from the web for off-line examination is undesirable and there is also high degree of sampling uncertainty associated with using a point sample to represent a large area.

In a different approach, Stanger et al. [21] used a weighing method to study the relationship between mass deposition rate for a whole mat, and electric current on the applied voltage. In the study, the mass of deposited fibre was found to be linear with deposition time. However, the measured masses of the fibres were only in the range of a few micrograms. Therefore, the measurement values were very small compared to the weight of the substrates used in the experiment, and at the limit of the four-figure balance used to take the readings. The balance precision is of particular relevance, as the surface and intrinsic charge on the collected fibres may well be enough to affect the mass readings obtained. The variation of area mass of the substrate that is typically part of the product for say a Petryanov type filter [22], will therefore have a highly significant effect on the process capability of results obtained from this type of method.

Image analysis could offer a non-destructive method for characterizing electrospun fibres [23], [24]. Semnani et al. [23] used image analysis based on grey scale image to determine the porosity of a nanofibres mat. Ziabari et al. [24] used image analysis to measure the diameters of electrospun nanofibres at the intersection point. Stanger et al. [25] validated an automated SEM image analysis system against manual techniques. Automating measurements allows a large number of images to be analysed in great detail, thus making it easier to make a truly representative estimate of complete product fibre size. Using digital images as inputs, image analysis has demonstrable potential to be used as a non-contact method for fibre structure analysis.

The objective of this study is to investigate the feasibility of using grey scale image analysis for predicting the amount of deposited electrospun nanofibres. It is important to note that this study is not about predicting the suitable amount of deposited fibres that suits a certain application, nor it is about optimization of the deposited amount. However, in this study a new method is proposed which will able to differentiate between products that have different amount of nanofibres. In addition, a comparison between image analysis and weighing methods is also conducted. A successful implementation of this study would 
provide a fundamental basis for a new and effective method for predicting the amount of deposited electrospun nanofibres.

\section{METHODS AND MATERIALS}

\section{Materials}

Poly(vinyl alcohol) (PVA) with an average molecular weight $\left(\mathrm{M}_{\mathrm{w}}\right)$ of 124,000-130,000 g/mol and degree of hydrolysis (DH) in the range of $86-89 \%$ was purchased from Polyscientific Enterprise Sdn. Bhd, Malaysia. A similar materials preparation procedure was used as described by a previous study [21]. The polymer solution with final concentration of $8 \mathrm{wt} \%$ was prepared by dissolving PVA in distilled water. The solution was stirred approximately for 2 hours at $60{ }^{\circ} \mathrm{C}$ using a hot plate magnetic stirrer Model C-MAG HS7 (Ika Works, Malaysia).

\section{Electrospinning Apparatus}

All experimental work was performed using a Model ES1a electrospinning machine (Electrospinz Ltd. New Zealand). The applied voltage was set a constant $10 \mathrm{kV}$ and the electrospinning distance was set at $10 \mathrm{~cm}$. The spinneret used in this experiment was an Axygen T-200-Y $200 \mu \mathrm{L}$ pipette tip with an orifice diameter of $0.5 \mathrm{~mm}$.

\section{Scanning Electron Microscope}

The morphology of the nanofibres was observed under scanning electron microscope (SEM) Model JEOL JSM-6010PLUS/LV. The samples were sputtered with platinum for 180 seconds using JEOL JEC-300FC auto fine coater. From the SEM micrographs, the average diameter of the nanofibres were measured using ImageJ software (National Institutes of Health, NIH, USA).

\section{The Weighing Method}

The weighing method was conducted similar to the method used by Stanger et al. [21] (Figure 2). Model AG204 four figure balance (Mettler Toledo, Switzerland); with measurement error of $\pm 0.0001 \mathrm{~g}$ was used throughout the experiment. Black A4 size papers $\left(80 \mathrm{~g} / \mathrm{m}^{2}\right.$, Kaskad Raven Black 1516RB) were used as the collection substrates to aid visibility. Prior to electrospinning sample, every piece of the blank substrate was weighed and recorded as initial weight, $w_{i}$. The weighing process was repeated at least three times to minimize error. All measurements were within 3 standard deviations of the calculated mean value. Then, the samples were produced by depositing electrospun nanofibres onto the substrates via normal electrospinning process. Triplicate samples were collected at different deposition times ranging from 30 seconds to 1800 seconds. After sample collection process, samples were left overnight to ensure that the solvent has fully evaporated. Then, the samples were weighed again using the similar procedure and the data was recorded as final weight, $w_{f}$. Finally, the amount of deposited electrospun nanofibres was calculated by subtracting the initial weight $w_{i}$ from the final weight $w_{f}$ (Weight of deposited nanofibres $=w_{f}-w_{i}$ ). 


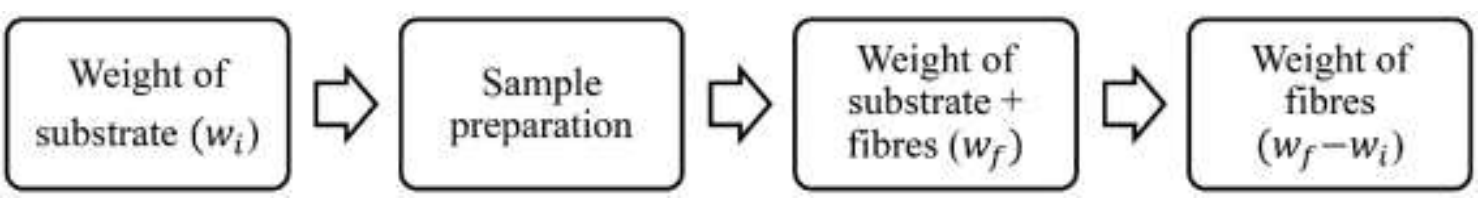

Figure 2. The sample weighing procedure.

\section{The image analysis method}

The image analysis process was conducted in a fixed step by step procedure as shown in Figure 3. The procedure was similar to the one used in a previous study [26]. After sample preparation process, samples were scanned into 8-bit grey scale images using a commercial Canon Model MG5500 scanner. The scanning resolution was set constant at 300 dots per inch (dpi). The scanned images were analyzed under constant settings using ImageJ software. Analysis area or region of interest (ROI) was selected using the rectangular selection tool in ImageJ software. The size of the ROI was about $8 \times 8 \mathrm{~cm}$ over the deposition area. The grey scale intensity of each pixel within the ROI were measured by the software and the built-in three-dimensional reconstruction tool in ImageJ software was used to construct topographic plot of the samples. Finally, the data were exported for statistical analyses. Regression analysis, probability value ( $\mathrm{p}$-value), Pearson correlation coefficient and t-test value were used to justify the results.

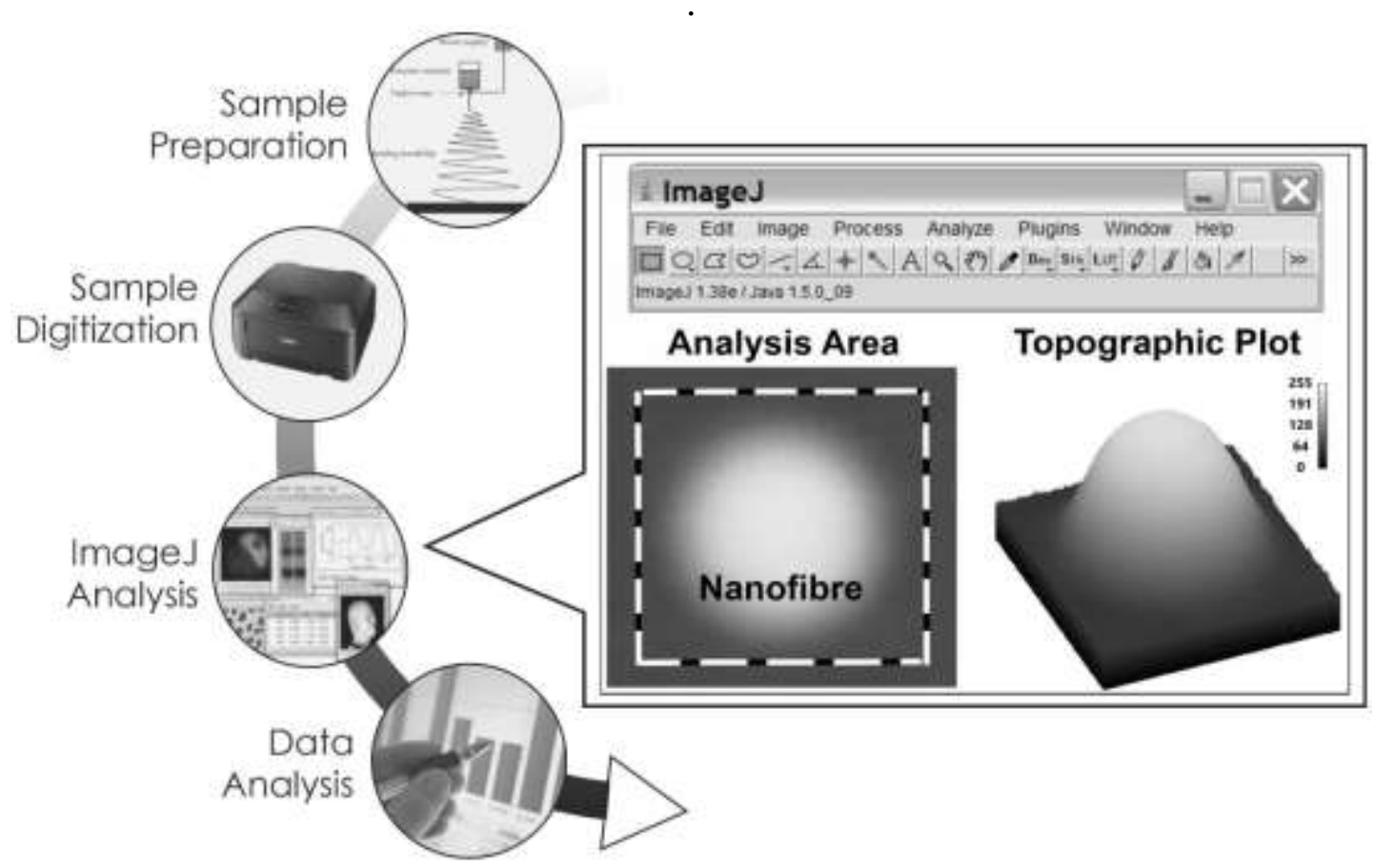

Figure 3. Image analysis procedure.

\section{Evaluation on different polymer and colored substrate materials}

Different polymer materials and coloured substrate materials were used to further test the capability of the image analysis method. In the first test, polyethylene oxide (PEO) (Sigma Aldrich 372773) at $6 \mathrm{wt} \%$ in water and Nylon 6 (Sigma Aldrich 181110) at $20 \mathrm{wt} \%$ in formic acid were used in this test. In the second test, the image analysis method was also tested by 
using different coloured paper substrates i.e. red, green, blue, orange and white. Electrospinning parameters such as applied voltage, tip to collector distance and deposition time were kept constant. In these test, the grey scale intensities across the substrate materials were measured. The graphs of grey scale intensity as a function of position were plotted.

\section{RESULTS AND DISCUSSION}

During the electrospinning process, the deposited nanofibres formed a round white spot on the collection substrate. From the sample, it was visually obvious that the size and the intensity of the white spot increased with the deposition time. As expected, a longer deposition time causes thicker fibre accumulation on the substrate resulting in a thicker and slightly larger deposition area. However, the size of the spot reached maximum size regardless of how long the deposition process was continued. This was because the size of the spot was limited by the size of the deposition cone formed during electrospinning process. A closer examination also revealed that within a deposition area, the central area seemed to be thicker compared to the edges. This suggests that more nanofibres accumulated at the central area compared to the edges. This finding was consistent with a previous study by Lee et al. [27], and likely to cause a problem if only small areas of deposition are examined, as for example with microscopic techniques.
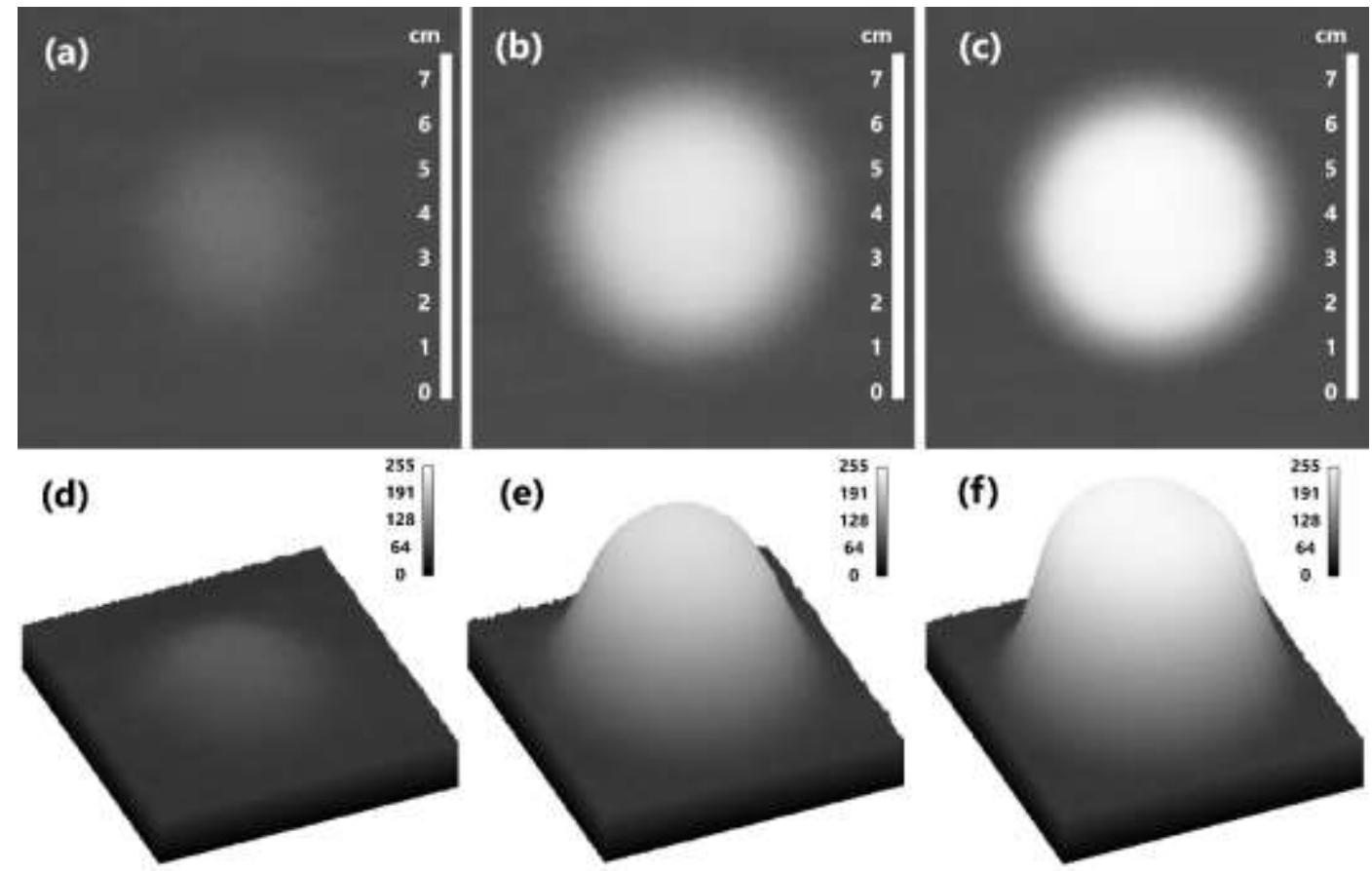

Figure 4. Deposited electrospun nanofibres collected at different deposition times (a) 30s

(b) $600 \mathrm{~s} \mathrm{(c)} \mathrm{1800s.} \mathrm{Subsequent} \mathrm{topographic} \mathrm{plots} \mathrm{of} \mathrm{grey} \mathrm{scale} \mathrm{intensities} \mathrm{of} \mathrm{the} \mathrm{samples} \mathrm{(d)}$ 30s (e) 600s (f) 1800s. 
From the scanned images of the samples in Figure 4 (a)-(c), topographic plots of grey scale intensities were made using ImageJ software (Figure 4 (d)-(f)). The samples were illustrated as three dimension (3D) bell-shaped profiles with binary representation of 0 for black and 255 for white for an 8-bit grey scale image. The shape of the plotted profiles confirmed the observations mentioned earlier. With reference to the scale bar, the height of the profile was observed to increase with deposition time. Furthermore, the bell-shaped profiles also proved that there were more nanofibres accumulated at the central area compared to the edges. This finding suggests that the expanding helix movement of fibres during electrospinning was not the perfect 'whipping' movement as commonly described in literature [28].

Plotting the peaks values of the 3D profiles against deposition time produced a plot as shown in Figure 5. In general, the grey scale value increased as the deposition time increased. Initially, the increment of grey scale values over time appeared to be in linear trend. However, the curve flattens when the greyscale value reached around 200 before reaching its maximum value of 255 . This was caused by the limitation of the greyscale shades available for an 8-bit greyscale image. It was noted that there was a slight jump in grey scale intensity value between $500 \mathrm{~s}$ to $750 \mathrm{~s}$ which could be due to common sources of variability during the experiment. However, this variation is thought insignificant because it did not affect the general trend of the data. It is also worth noting that the curve did not start at zero. This is because the baseline was found to be around 50 which was determined by measuring the greyscale values of blank substrates.

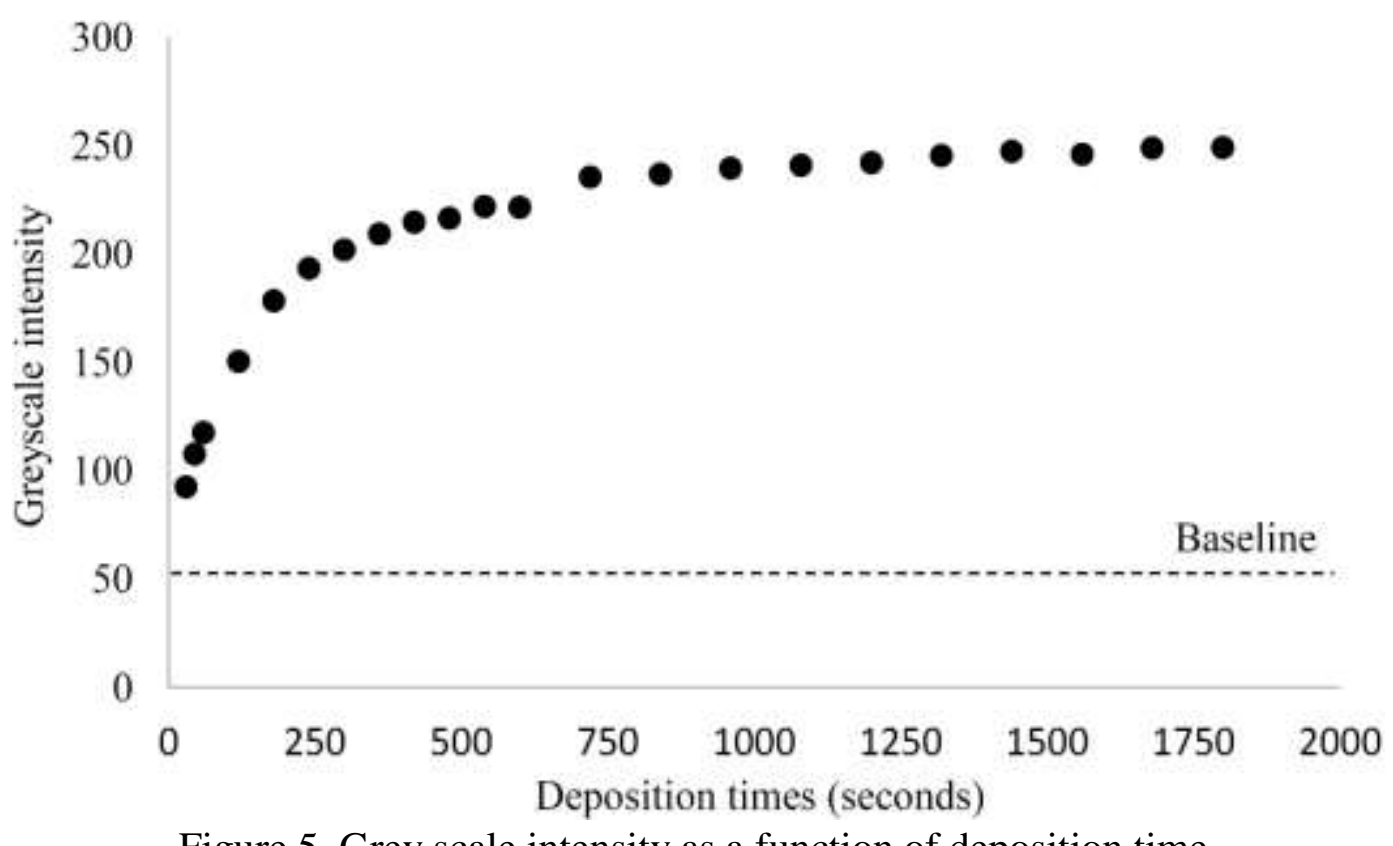

Figure 5. Grey scale intensity as a function of deposition time.

As a comparison, the same set of the samples were analysed using weighing method. A plot of fibre masses as a function of deposition time was made as shown in Figure 6. Similar to image analysis method, the amount of electrospun fibres increased as the deposition time increased. About $95 \%\left(\mathrm{R}^{2}=0.9475\right)$ of the variability of mass of fibre is explained by the deposition times. This results corroborates the finding of a previous study by a Stanger et al. 
[21] which suggested that for a given set of production parameters, the mass of deposited electrospun nanofibres is proportional to deposition time. However, measurements of deposited fibre masses were observed to be scattered at short deposition times especially at below 200 seconds (Figure 6). This result might be explained by the fact that the calculated standard deviation of the substrates mass was high $( \pm 0.0537 \mathrm{~g})$ compared to the actual mass of the deposited fibres (0.02-0.03 g). The y-intercept at $\mathrm{t}=0$ is not a zero mass due to residual electrostatic charge in the fibres affecting the balance readings as quantified by Stanger et al. [21].

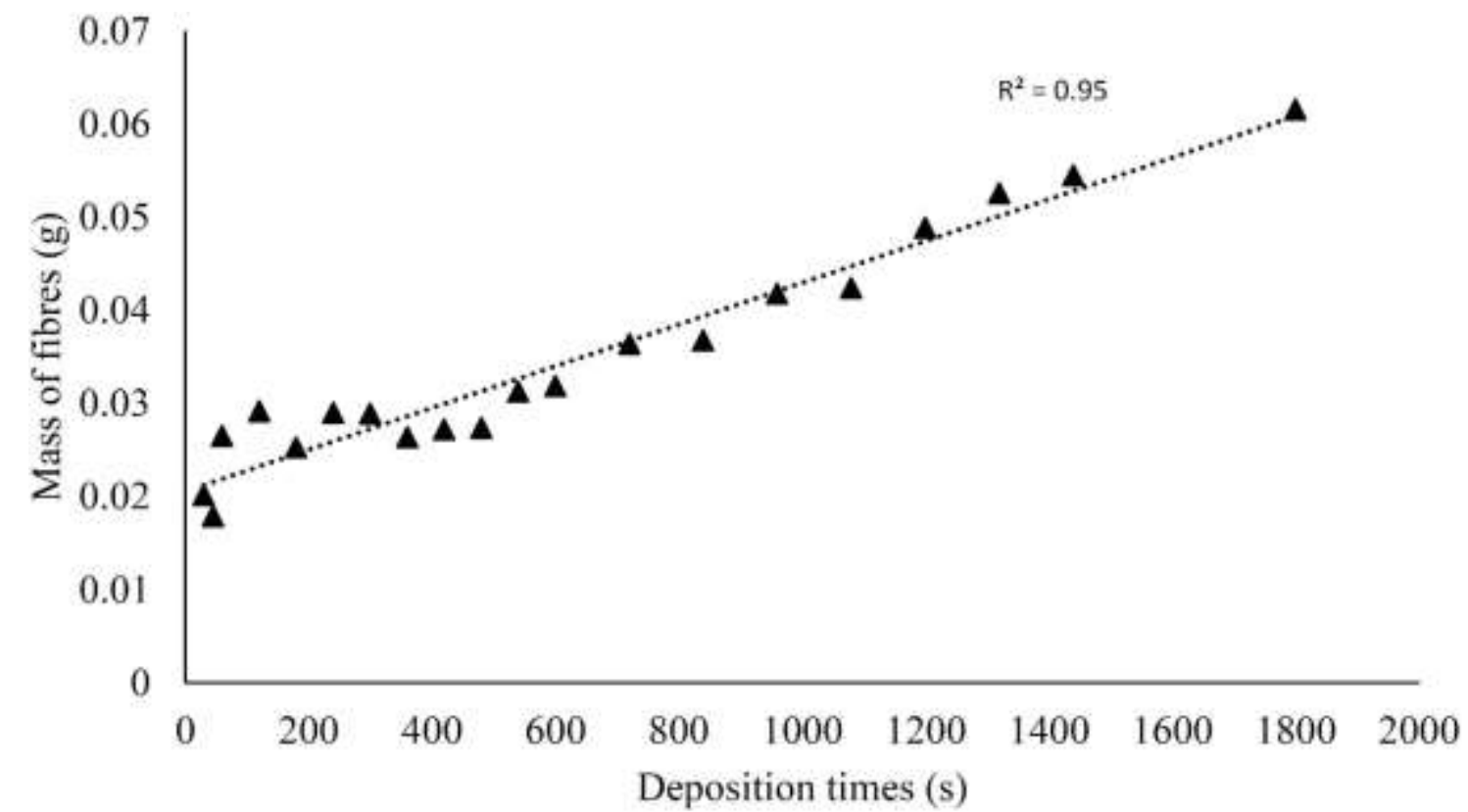

Figure 6. Mass of fibres as a function of deposition time.

Linear regression analysis was used to compare the results between image analysis and weighing methods at short deposition times as shown in Figure 7. Statistical measurements such as Pearson correlation, coefficient of determination and t-test were used to compare the results as summarize in Table 1. A best fit line was fitted to each set of the data for deposition times of 30 to 180 seconds. The coefficient of determination $\left(\mathrm{R}^{2}\right)$ value for image analysis method and weighing method were $98.9 \%$ and $36.4 \%$ respectively. The results suggest that the fitted model for image analysis data explains $98.9 \%$ of the variability of the data around its mean compared to only $36.4 \%$ for weighing method.

$\mathrm{P}$-values were used to test the significance of the data towards the deposition time. In this case, the null hypothesis is that there is no significant difference between the sample mean of grey scale intensity and mass of fibres. The images analysis data showed a greater evidence against the null hypothesis ( $\mathrm{p}$-value 0.0001 ) compared to the weighing data (p-value 0.2821 ). From the results, it is safe to say that at short deposition times, the fitted model for image analysis method was in better agreement with the data compared to the weighing method. In addition, the plot shows a strong positive linear correlation between grey scale intensity and deposition time. There is an encouraging finding that when using the Pearson correlation coefficient to measure the relationship between dependent and independent variables for both methods, a strong positive relationship of image analysis method was 
obtained with $r$ value of 0.99431 . The $r$ value for weighing method was 0.0140 . Further statistical tests revealed that a positive correlation between grey scale intensity and deposition time was obtained when using image analysis method. This was further supported by t-test values for image analysis and weighing method of 16.1672 and 1.3043 respectively. The larger value of t-test indicates that there is a significant relationship between greyscale intensity and deposition time.

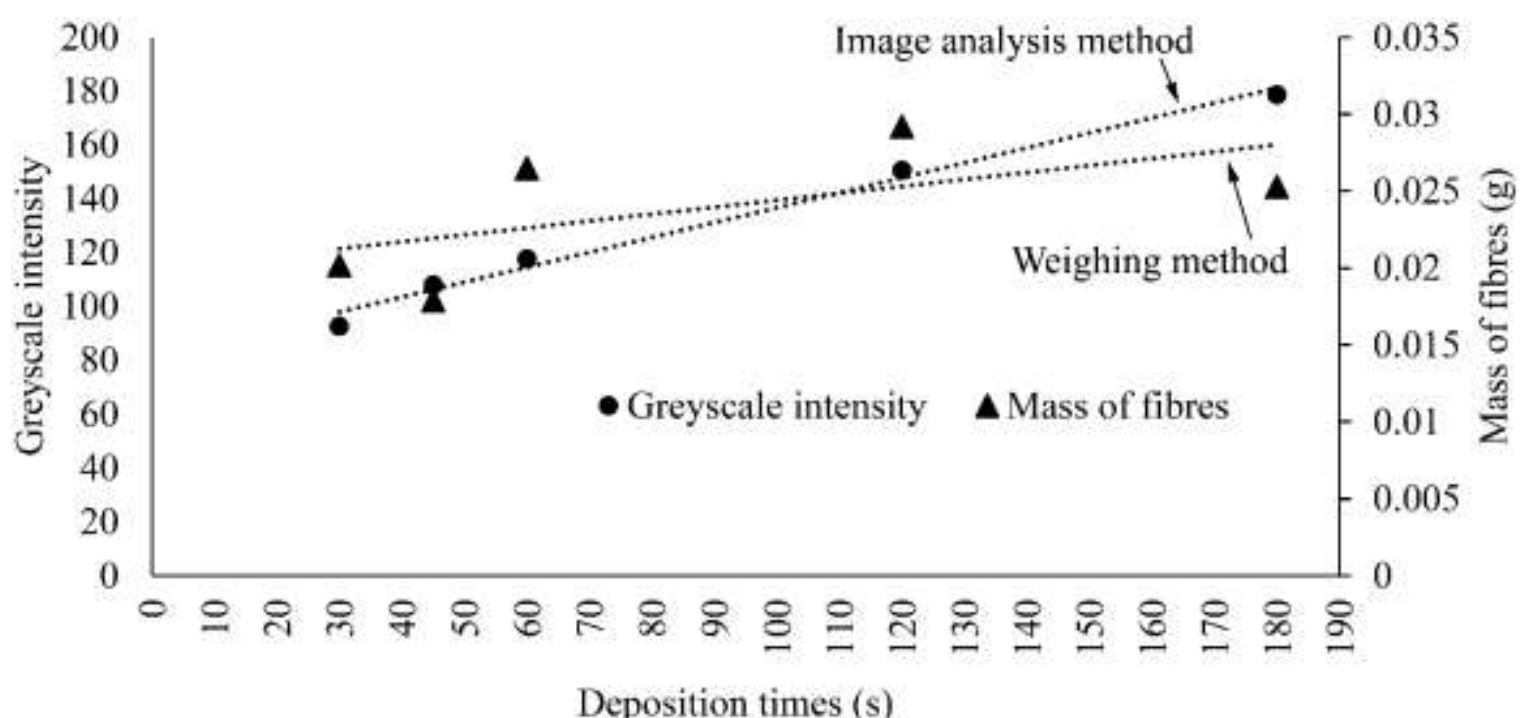

Figure 7. A comparison between image analysis method and weighing method at short deposition times of 30 to 180 s.

Table 1. A summary of data from statistical analysis

\begin{tabular}{lll}
\hline & Weighing technique & Image analysis technique \\
\hline General equation & $\mathrm{y}=5 \mathrm{E}-0.5 \mathrm{x}+0.02 \mathrm{~s}$ & $\mathrm{y}=0.555 \mathrm{x}+81.26$ \\
$\left(\mathrm{R}^{2}\right)$ & $36.4 \%$ & $98.9 \%$ \\
$\mathrm{p}$-value & 0.2821 & 0.0001 \\
$\mathrm{r}$ & 0.0140 & 0.9943 \\
$\mathrm{t}$-test & 1.3043 & 16.1672 \\
\hline
\end{tabular}

Different materials were used to further test the capability of the image analysis method. Using the same procedures as described earlier, the grey scale intensities of the samples were plotted as shown in Figure 8. The results suggest that the image analysis method could be applicable to other materials. The grey scale intensities of PEO and Nylon 6 were identical to PVA where the central area seemed to be thicker compared to the edges. However, it was also found that the shape and peak values of the plots were different. This could be explained by the fact that each material has its own unique electrospinning characteristics such as the formation of the electrospinning cone and mass deposition rate. 


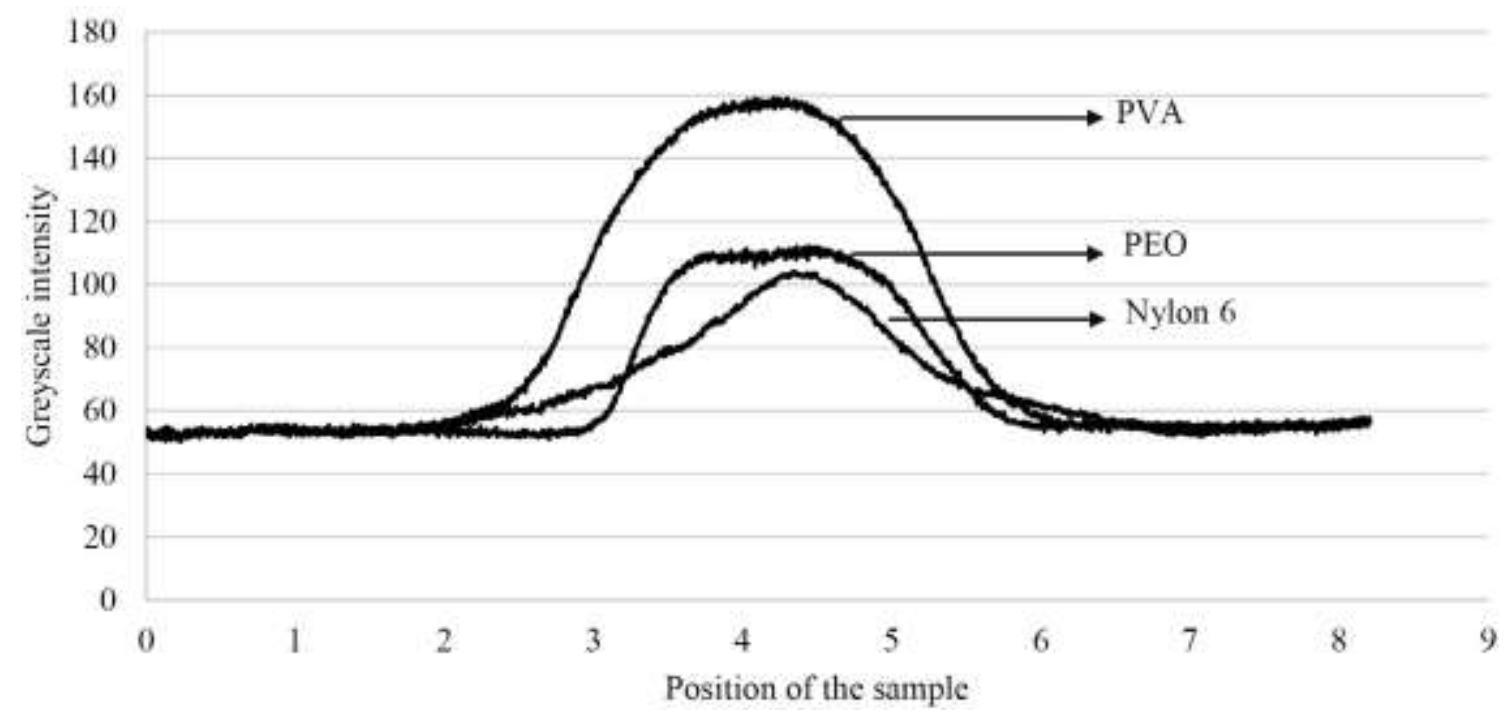

Figure 8. Grey scale intensity profile with different materials.

The image analysis method was also tested by using different coloured paper substrates i.e. red, green, blue, orange and white. Plots of grey scale intensities of the samples are shown in Figure 9. As expected, the baselines of other coloured substrates were higher compared to the black substrate. This was because the grey scale algorithms used by the scanner converted the colour papers into a higher shades of grey depending on the original pixel values of the colour in RGB colour space [29]. Since the image analysis method used relies on the contrast between the deposited web area and the background, using a bright substrate paper limits the capability of the method as evidenced in Figure 9. Thus, for the best results, a dark substrate is preferable when using this method.

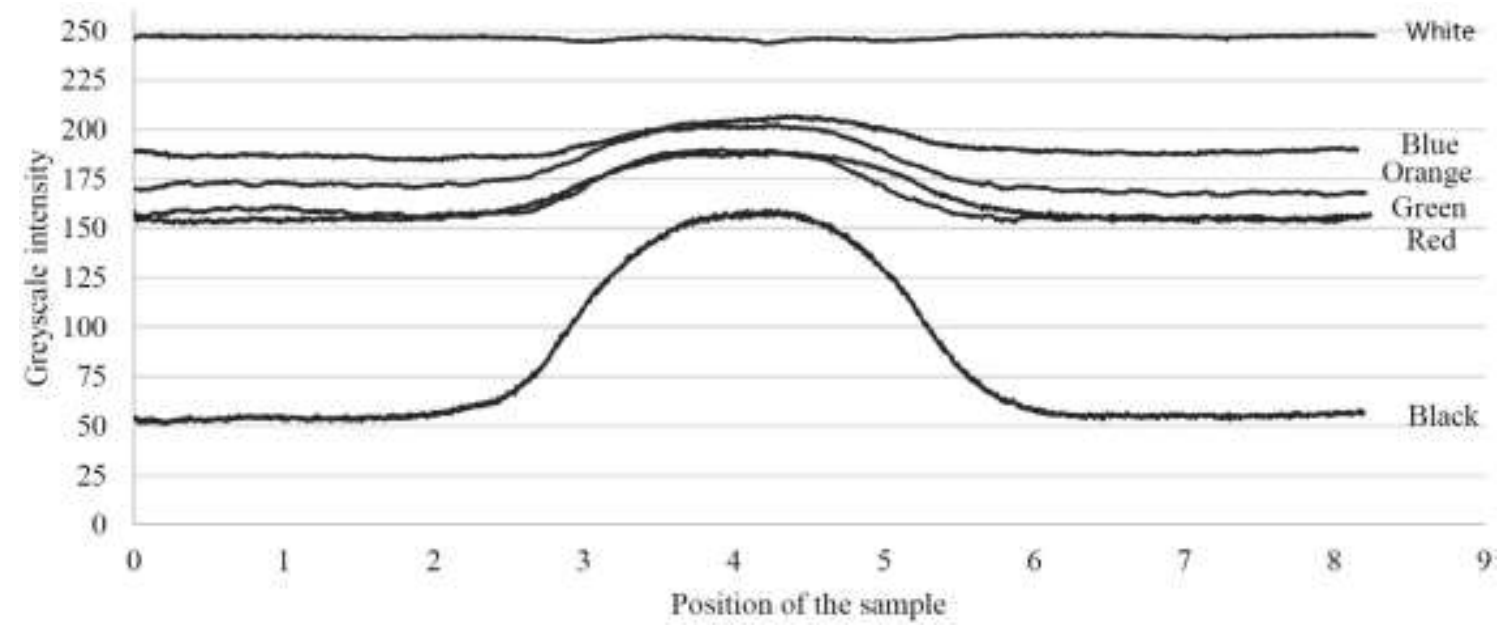

Figure 9. Grey scale intensity profile with different substrates. 
From scanning electron micrographs, uniform randomly orientated PVA electrospun fibres were observed with no sign of abnormalities such as beading or ribbon formation (Figure 10). Fibre diameters were measured at 100 different locations to minimize error. The measured average fibre diameter was $231.71 \mathrm{~nm}( \pm 17.15 \mathrm{~nm})$ as shown in Figure 11. The appearance of the electrospun fibres was normal and the diameters were within the typical fibre diameter of the material used. This suggests that the use of paper substrates did not interfere with the formation, thinning and solidifying process of the electrospun nanofibres.

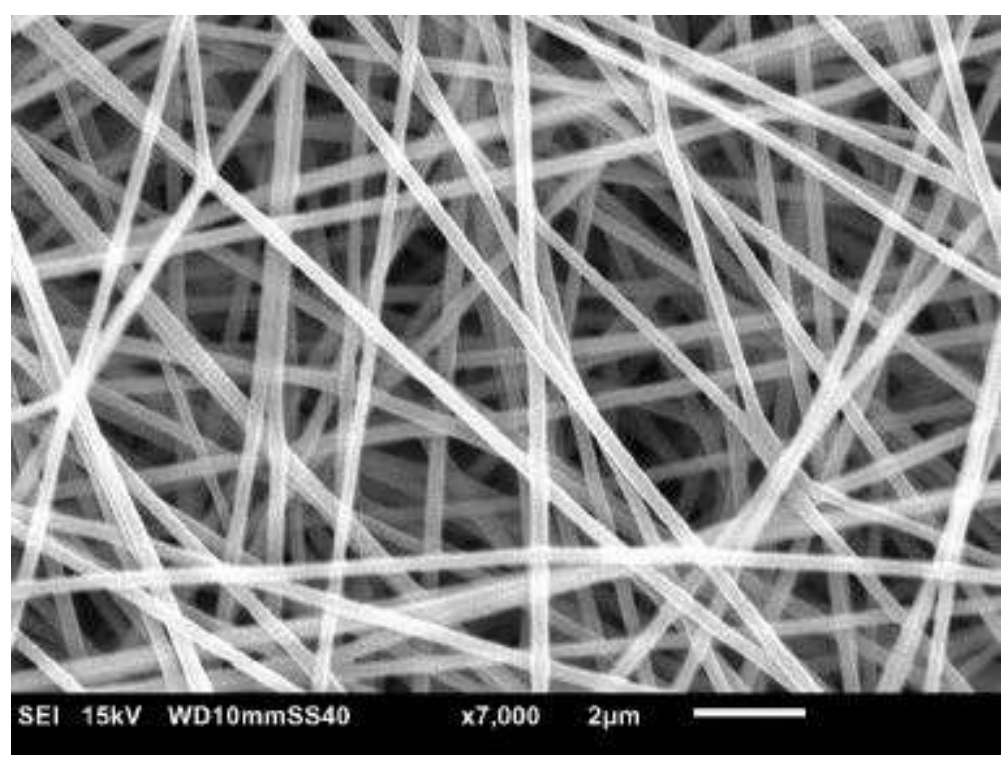

Figure 10. A typical scanning electron micrograph of PVA electrospun nanofibres.

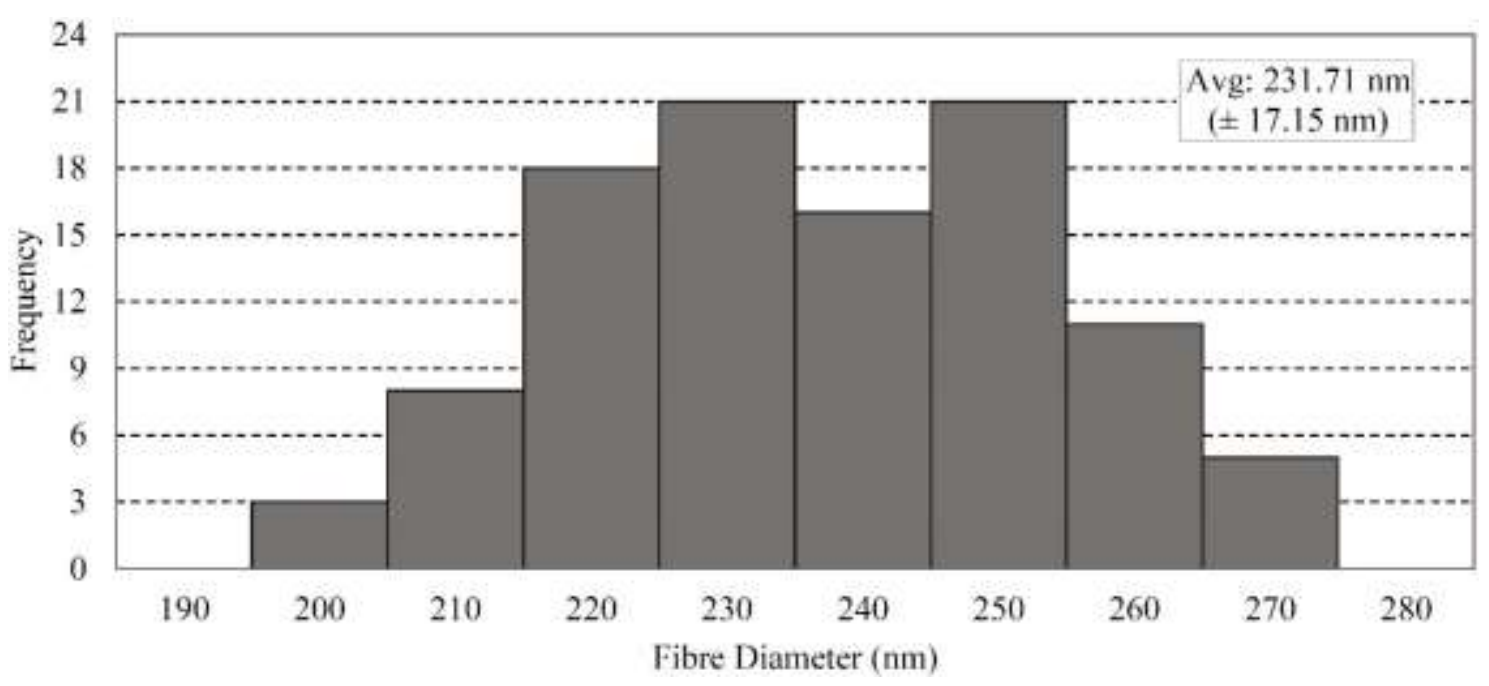

Figure 11. A histogram showing the measured diameters of the electrospun nanofibres. 
The results of this study provide a basis of understanding for applying large area image analysis to predict the amount of deposited electrospun nanofibres. The proposed method has the potential to be used for process control in electrospun fibre production. The main advantages of this method are; (i) the method can be reliably used to measure the smallest amount of nanofibres even when a conventional weighing method is incapable of providing reliable data (ii) the method can be automated using a non-contact scanner providing a quick and non-destructive in-line quality control method.

Despite the clear potential, demonstrated by the image analysis method, the following limitations should be noted. First, when using this method to determine the amount of deposited electrospun nanofibres, a linear correlation between grey scale intensity and deposition times is preferred. However, as shown in Figure 5, the correlation was not entirely linear. This is due to the limitation of an 8-bit image which only allows 256 level of intensities. This can be improved by using a deeper pixel depth format such as 16-bit grey scale images. Second, this method is currently correlates grey scale intensities and deposition times. However, an estimation of deposited mass can also be done by establishing a correlation between grey scale intensities and masses of the deposited fibres. Further studies on these limitations are ongoing and the results will be reported later.

\section{CONCLUSION}

The findings of this study show that the grey scale image analysis method was a better option compared to the weighing method, especially for determining a small amount of deposited electrospun nanofibres. In general, both methods were capable of showing the increasing amount of deposited nanofibres with respect to time. However, a direct comparison between the two methods showed that image analysis method was superior at short deposition times, which are often required in commercial production. Statistical analyses confirmed that a strong correlation was found between grey scale intensities and deposition times, compared to a weaker correlation when using weighing method. The coefficient of determination $\left(\mathrm{R}^{2}\right)$ value for image analysis method was $98.9 \%$ (p-value 0.0001 ) compared to only $36.4 \%$ (pvalue 0.2821 ) for weighing method. This was because at short deposition times the measured nanofibres masses were insignificant compared to the weight variation of the substrates. The findings of this study indicate clear potential for a new non-destructive method for predicting the amount of deposited electrospun fibres, because we have demonstrated that image analysis is a non-destructive technique capable of quantifying deposition over large areas of fibre.

\section{ACKNOWLEDGEMENTS}

The authors would like to thank the Ministry of Higher Education Malaysia and the Universiti Teknikal Malaysia Melaka (UTeM) for sponsoring this project through the Fundamental Research Grant Scheme FRGS/2/2014/TK01/FKM/02/F00233. Special thanks to the members of Advanced Materials Research Group (A-MAT), Centre for Advanced Research on Energy (CARe), and the Faculty of Mechanical Engineering, UTeM for their continuous support for this project. 


\section{REFERENCES}

[1] Huang ZM, Zhang YZ, Kotaki M, Ramakrishna S. A review on polymer nanofibers by electrospinning and their applications in nanocomposites. Composites Science and Technology 2003; 63: 2223-2253.

[2] Teo WE, Ramakrishna S. A review on electrospinning design and nanofibre assemblies. Nanotechnology 2006; 17(14): R89-R106.

[3] Chronakis IS. Novel nanocomposites and nanoceramics based on polymer nanofibers using electrospinning process - A review. Journal of Materials Processing Technology 2005; 167: 283-293.

[4] Tucker N, Stanger JJ, Staiger MP, Razzaq H, Hofman K. The history of the science and technology of electrospinning from 1600 to 1995. Journal of Engineered Fibers and Fabrics. Special Fibers Edition 2012; 7(2): 63-73.

[5] Taylor G. Disintegration of water drops in an electric field. Proceedings of the Royal Society A - Mathematical, Physical and Engineering Sciences 1964; 280(1382): 383397.

[6] Zhang C, Yuan X, Wu L, Han Y, Sheng J. Study on morphology of electrospun poly(vinyl alcohol) mats. European Polymer Journal 2005; 41: 423-432.

[7] Reneker DH, Yarin AL, Fong H, Koombhongse S. Bending instability of electrically charged liquid jets of polymer solutions in electrospinning. Journal of Applied Physics 2000; 87(2000): 4531-4547.

[8] Ramakrishna S, Fujihara K, Teo WE, Yong T, Ma Z, Ramaseshan R. Electrospun nanofibers: Solving global issues. Materials Today. 2006; 9(3): 40-50.

[9] Lu P, Ding B. Applications of electrospun nanofibers. Recent Patents on Nanotechnology 2008; 2(3): 169-182.

[10] Matsumoto H, Tanioka A. Functionality in electrospun nanofibrous membranes based on fiber's size, surface area, and molecular orientation. Membranes (Basel) 2011; 1: 249-264.

[11] Owoeye FT, Azodo AP, Udo SB. Performance evaluation of ceramics microfiltration membrane for water treatment. Journal of Mechanical Engineering and Sciences 2014; 7: 1115-1126.

[12] Said KAM, Jamain RL, Sutan NSM, Alipah NAM. Enhanced permeation performance with incorporation of silver nitrate onto polymeric membrane. Journal of Mechanical Engineering and Sciences 2018; 12(3): 3811-3824.

[13] Roslan NSA, Abdul Hamid N, Md Isa MH, Muhammad N, Mansor MR, Abdul Munajat N. Nylon electrospun nanofibre water filtration media for wastewater treatment. Materials Research Express 2018; 5(10): 105010.

[14] Gibson P, Schreuder-Gibson H, Rivin D. Transport properties of porous membranes based on electrospun nanofibers. Colloids and Surfaces A: Physicochemical and Engineering Aspects 2001; 187-188: 469-481.

[15] Lee S, Obendorf SK. Use of electrospun nanofiber web for protective textile materials as barriers to liquid penetration. Textile Research Journal 2007; 77(9): 696-702.

[16] Li J, Gao F, Liu LQ, Zhang Z. Needleless electro-spun nanofibers used for filtration of small particles. Express Polymer Letters 2013; 7(8): 683-689. 
[17] Aussawasathien D, Teerawattananon C, Vongachariya A. Separation of micron to sub-micron particles from water: Electrospun nylon-6 nanofibrous membranes as prefilters. Journal of Membrane Science 2008; 315: 11-19.

[18] Barhate RS, Loong CK, Ramakrishna S. Preparation and characterization of nanofibrous filtering media. Journal of Membrane Science 2006; 283(1-2): 209-218.

[19] Leung WWFF, Hung CHH, Yuen PTT. Effect of face velocity, nanofiber packing density and thickness on filtration performance of filters with nanofibers coated on a substrate. Separation and Purification Technology 2010; 71(1): 30-37.

[20] Affandi NDN, Truong YB, Kyratzis IL, Padhye R, Arnold L. A non-destructive method for thickness measurement of thin electrospun membranes using white light profilometry. Journal of Materials Science 2010; 45: 1411-1418.

[21] Stanger J, Tucker N, Fullick S, Sellier M, Staiger MP. Insights into the power law relationships that describe mass deposition rates during electrospinning. Journal of Materials Science 2012; 47(3): 1113-1118.

[22] Filatov Y, Kirichenko V, Budyka A. Electrospinning of micro and nanofibers: Fundamentals and applications in separation and filtration processes. New York: Begell House; 2007.

[23] Semnani D, Ghasemi-Mobarakeh L, Morshed M, Nasr-Esfahani MH. A novel method for the determination of cell infiltration into nanofiber scaffolds using image analysis for tissue engineering applications. Journal of Applied Polymer Science 2009; 111(1): 317-322.

[24] Ziabari M, Mottaghitalab V, McGovern ST, Haghi AK. A new image analysis based method for measuring electrospun nanofiber diameter. Nanoscale Research Letters 2007; 2: 597-600.

[25] Stanger JJ, Tucker N, Buunk N, Truong YB. A comparison of automated and manual techniques for measurement of electrospun fibre diameter. Polymer Testing 2014; 40: 4-12.

[26] Che Long F, Nurfaizey AH, Daud MAM. A preliminary study of greyscale intensity and deposited electrospun fibres using image analysis technique. Proceedings of Mechanical Engineering Research Day 2016; 165-166.

[27] Lee J, Jeong YH, Cho DW. Fabrication of nanofibrous mats with uniform thickness and fiber density. Macromolecular Materials and Engineering 2014; 299: 1052-1061.

[28] Nurfaizey AH, Stanger J, Tucker N, Buunk N, Wood AR, Staiger MP. Control of spatial deposition of electrospun fiber using electric field manipulation. Journal of Engineered Fibers and Fabrics 2014; 9(1): 155-164.

[29] Benedetti L, Corsini M, Cignoni P, Callieri M, Scopigno R. Color to gray conversions in the context of stereo matching algorithms. Machine Vision and Applications 2010; 57(2): 254-348. 